\title{
围封对温带半干旱典型草原群落种间关联的影响
}

\author{
龚 容 ${ }^{1,2}$ 高 琼 ${ }^{1,2 *}$ 王亚林 ${ }^{1,2}$
}

${ }^{1}$ 北京师范大学地表过程与资源生态国家重点实验室, 北京 100875 $^{2}$ 北京师范大学减灾与应急管理研究院, 北京 100875

摘 要 为明确围栏封育对温带典型草原群落物种种间关系的影响, 运用方差比率法、基于 $2 \times 2$ 列联表的 $\chi^{2}$ 检验、Ochiai指 数、Spearman相关分析, 对位于内蒙古锡林郭勒盟太仆寺旗封育6年的围封样地及围栏外样地的主要优势种及伴生种进行了 种间关联和相关性分析。同时运用生态位宽度指数分析围栏内外物种对环境资源的利用能力及种间竞争情况。结果表明: 1) 基于 $2 \times 2$ 列联表的 $\chi^{2}$ 检验结果表明围栏内 9 个种对显著正联结, 4 个种对显著负联结 $(p \leqslant 0.05)$, 围栏外有 19 个种对显著正联 结, 8 个种对显著负联结; Spearman秩相关分析表明围栏内有 10 个种对显著正相关, 16 个种对显著负相关, 围栏外有 12 个种对 显著正相关, 30 个种对显著负相关。 $\chi^{2}$ 检验和Spearman检验结果表明围栏内群落处于稳定发展状态, 而围栏外群落处于退化阶 段。2)围栏内外的优势种Ochiai指数都较高, 但 $\chi^{2}$ 检验并不显著, 表明优势种之间的关联程度高, 但存在相对独立的分布格局。 3)围栏内主要优势种的生态位宽度大于围栏外, 说明围栏封育保护了主要优势种的生长。

关键词 围栏育封; 重要值; 种间联结; 生态位宽度; 资源利用

引用格式: 枈容, 高琼, 王亚林 (2016). 围封对温带半干旱典型草原群落种间关联的影响. 植物生态学报, 40, 554-563. doi: 10.17521/cjpe.2015.0446

\section{Effects of exclosure on community inter-specific relationships in a typical temperate grassland} GONG Rong ${ }^{1,2}$, GAO Qiong ${ }^{1,2 *}$, and WANG Ya-Lin ${ }^{1,2}$

${ }^{1}$ State Key Laboratory of Earth Surface Processes and Resource Ecology, Beijing Normal University, Beijing 100875, China; and ${ }^{2}$ Academy of Disaster Reduction and Emergency, Beijing Normal University, Beijing 100875, China

\section{Abstract}

Aims This study aimed to understand the impact of exclosure on interspecific associations of species in a typical temperate grassland. We compared the species associations of dominant and companion species in grassland plots with the six-year exclosure and grazing in Taips County, Nei Mongol.

Methods We selected 14 species according to important values in each plot, using variance ratio to analyze the overall association. The $\chi^{2}$ test with $2 \times 2$ contingency table, Ochiai index and spearman rank correlation test were applied to analyze the inter-specific associations of dominant and companion species. We used the niche breadth index to analyze the ability of resource utilization of species and inter-specific competition for grazed plots and ones with exclosure.

Important findings The inter-specific associations among 91 species pairs inside the exclosure showed that nine species pairs were significantly positive but 4 pairs were negative based on the $\chi^{2}$ test with $2 \times 2$ contingency table. Ten species pairs had significantly positive correlations and 16 species pairs were negative through the Spearman correlation test. For the plots outside the exclosure, we found 19 species pairs were positively associated but 8 pairs were negative associated using the $\chi^{2}$ test, and 12 species pairs were significantly positively correlated but 30 pairs were negatively correlated using the Spearman correlation test. The results indicated that the community inside the exclosure was in a continuous development state while the outside community was degradating. The Ochiai indices of dominant species were high both inside and outside the exclosure but the differences using the $\chi^{2}$ test were nonsignificant. It implied that there was some connection among dominant species but their patterns of distribution were relatively independent. The niche breadth for dominant species inside the exclosure was higher than ones outside, which suggested the exclosure has an important role in protecting dominant species. Key words exclosure; importance value; interspecific association; niche breadth; resources utilization

Citation: Gong R, Gao Q, Wang YL (2016). Effects of exclosure on community inter-specific relationships in a typical temperate grassland. Chinese Journal of Plant Ecology, 40, 554-563. doi: 10.17521/cjpe.2015.0446

收稿日期 Received: 2015-12-07 接受日期 Accepted: 2016-04-23

* 通信作者Author for correspondence (E-mail: gaoq@bnu.edu.cn) 
目前，围栏封育是退化草地植被恢复的有效措 施, 它能够使沙化草地植物群落的组成发生规律性 的变化。而种间联结性分析是探讨群落稳定性与演 替过程中种间关系动态变化的有效途径(张明霞等, 2015)。种间联结是指不同物种在空间分布上的相互 关联性, 通常是由于群落生境的差异影响物种分布 而引起的。植物种对间的正联结和正相关体现了植 物利用资源的相似性和生态位的重叠性, 主要是由 于植物具有相近的生物学特性及对生境具有相似的 生态适应性(张峰和上官铁梁, 2000)。正相关的物种 可以提高单独生长不易存活物种的生长和存活率 (Callaway, 1996; Zaal \& Georgi, 1998), 对群落多样 性的维持及生态系统服务功能的发挥有很大的作用 (Callaway et al., 2002)。负联结和负相关体现了物种 间的排斥性, 这是物种长期适应不同微环境, 利用 不同空间资源的结果, 也是生态位分离的反映(李 新荣, 1999)。研究表明对有限资源的竞争是维持生 物多样性的主要压力(Nuñez et al., 1999; Palmer et al., 2003)。长期的竞争排除等生态过程作用使得种 群之间的演替产生了某种程度的生态位分离(刘小 丹等, 2015)。物种的生态学及进化生物学特征、种 间的相互作用关系、群落中物种分布的均匀程度、

放牧、火烧、围封等干扰因素都能影响物种生态位 宽度的大小(李军保等, 2009; 井光花等, 2015)。在放 牧影响下, 植物主要通过大幅度调节生态位宽度来 保证植物种群存活及其在群落中的地位和作用, 对 放牧耐受性较强的物种, 其生态位宽度变化较小 (吕世杰等, 2014)。李中林等(2014)的研究结果表明 围栏封育区的植物生态位宽度要高于自由放牧区的 生态位宽度。

徐粒等(2014)在内蒙古太仆寺旗围封样地上的 研究结果表明围栏内的地上生物量显著大于围栏 外, 而物种丰富度显著小于围栏外。植物个体的分 布格局以及不同演替阶段群落中物种之间的关系是 植物群落与环境之间长期作用的结果 (Nathan, 2006)。国内外许多学者基于不同植被类型的优势种 种间关系做了很多的研究(Henry, 1998; Grechanichenko, 2001; 刘珏宏等, 2010; 贾呈金卓等, 2014; 李兰兰等, 2014), 但是基于多年围栏封育对草原植 被种间关系的影响研究较少。本文旨在通过研究在 围封6年后围栏内外植物群落间种间关联的差异, 结合生态位宽度分析, 阐明围封对草原生态系统物
种生态位变化的影响, 揭示群落种间关系并了解群 落结构波动情况。

\section{1 材料和方法}

\section{1 研究区概况}

研究区位于内蒙古太仆寺旗(114.85-115.82 ${ }^{\circ}$ E, 41.58ㅇ- $42.17^{\circ} \mathrm{N}$ ), 属低山丘陵区, 地势起伏不平, 坡缓而谷宽, 海拔1 300-1 800 m。该区属中温带半 干早大陆性气候, 年平均气温 $2.1{ }^{\circ} \mathrm{C}$, 每年 1 月为最 冷月, 平均气温 $-18{ }^{\circ} \mathrm{C}$, 最低气温 $-36{ }^{\circ} \mathrm{C}$ 。7 月为最热 月, 平均气温 $21{ }^{\circ} \mathrm{C}$, 最高气温可达 $34{ }^{\circ} \mathrm{C}$ 。全年无霜 期110天左右。降水量主要集中在6、7、8 三个月。 近35年来年降水量384.5 mm。研究区地带性土壤为 栗钙土, 土壤有机质含量较低。太仆寺旗是典型的 农牧交错生态过渡区(邓祥征等, 2004), 属内蒙古克 氏针茅-羊草草原。草原植被主要有: 冷蒿(Artemisia frigida)、粘隐子草(Cleistogenes squarrosa)、克氏针 茅(Stipa krylovii)、羊草(Leymus chinensis)等。主要 伴生种有冰草 (Agropyron cristatum) 、花苜宿 (Medicago ruthenica)、寸草(Carex duriuscula)、阿尔 泰狗娃花(Aster altaicus)、细叶韭(Allium tenuissimum)、二裂委陵菜(Potentilla bifurca)、矮非(Allium anisopodium)等。另外有呈斑块状的小叶锦鸡儿 (Caragana microphylla)灌丛分布在围栏内外。

\section{2 野外实验设计}

2007年10月, 在太仆寺旗(位于内蒙古自治区 锡林郭勒盟, $42.07^{\circ} \mathrm{N}, 115.28^{\circ} \mathrm{E}$, 海拔 $1370 \mathrm{~m}$ )东北 黑山庙地区设置坡向为西北一东南向, 沿坡面从上 到下 $400 \mathrm{~m}$ 横向 $50 \mathrm{~m}$ 的围栏样地, 围栏内全年禁牧。 在隔围栏样地 $5 \mathrm{~m}$ 选取同样面积大小的平行坡面作 为对照样地。该区在2003年以前重度放牧至退化阶 段, 2003年以后轻度放牧, 每年的生长季末期(9月 中下旬)会有打草活动。2013年7月, 将围栏样地等 分为 20 个 $20 \mathrm{~m} \times 50 \mathrm{~m}$ 的研究小区。在每个研究小区 内随机选取 5 个草本样方 $(1 \mathrm{~m} \times 1 \mathrm{~m})$, 用针刺法调 查草本植物的种类、高度和分盖度等。围栏内外总 计 200 个样方。在调查的 200 个样方中共出现 37 个物 种, 在围栏内外分别选取重要值大于 2 的前 14 个物 种进行种间关联性分析(柳剑丽, 2013)。

\section{3 数据分析方法}

将群落中物种在各样方中出现的频度进行统 计, 根据出现的频度排除偶见种, 把主要成对物种 
的定性数据列入 $2 \times 2$ 列联表, 物种在各样方内的出 现情况作为原始数据进行分析, 计算种间联结性, 并进行 $\chi^{2}$ 检验和各种对间的Ochiai指数的计算, 同 时利用方差比率法计算草本群落的整体联结性。应 用定量数据(重要值)对种间关系进行Spearman秩相 关分析(张金屯, 2004)。

\subsection{1 重要值的计算}

重要值 $(I V)$ 是表征某个物种在群落中的地位和 作用的综合数量指标。

$I V=($ 相对盖度 + 相对高度 + 相对频度 $) / 3$

\subsection{2 总体关联性检验}

群落的总体关联性能反映出群落的稳定性, 体 现出物种的空间分布关系以及物种间的功能。

Schluter提出的方差比率 $(V R)$ 可以检验多个物种间 总体关联的显著性。

$$
V R=\frac{S_{T}^{2}}{\delta_{T}^{2}}
$$

其中, 总样本方差 $\delta_{T}^{2}=\sum_{i=1}^{\mathrm{s}} P_{i}\left(1-P_{i}\right)$, 总种数方差 $S_{T}^{2}=\frac{1}{N} \sum_{i=1}^{s}\left(T_{j}-t\right)^{2}, \quad P_{i}=\frac{n_{i}}{N}, \quad t=\frac{T_{1}+T_{2}+\ldots T_{N}}{N}$, $T_{j}$ 为第 $j$ 样方物种总和, $P_{i}$ 指某物种出现的样方数占 总样方数的比例, $n_{i}$ 为第 $i$ 物种出现的样方数, $N$ 为样 方总数。 $V R$ 在独立零假设的条件下其期望值为 $1, V R$ $=1$, 说明所有物种种间无关联, $V R>1$, 说明种间 表现出正联结, $V R<1$ 则表现出负联结。 $\omega(\omega=V R \times$ $N)$ 是检验 $V R$ 显著程度的统计量, 若 $\omega$ 落入 $\chi^{2}$ 分布给 出的界限内 $\left.\chi^{2}{ }_{0.95}(N)<\omega<\chi_{0.05}^{2}(N)\right)$, 则种间关联不 显著。

\subsection{3物种种间关联分析}

基于 $2 \times 2$ 列联表的 $\chi^{2}$ 统计量, 可以检验种对间 的联结显著性。由于取样的非连续性, 常常会造成 偏低估计, 这种偏差可以用Yates的连续校正系数来 校正，其公式为:

$$
\chi^{2}=\frac{N[|a d-b c|-0.5 N]^{2}}{(a+b)(a+c)(c+d)(b+d)}
$$

式中 $a$ 表示存在有 $\mathrm{A} 、 \mathrm{~B}$ 两个物种的样方数, $b$ 表示物 种 $\mathrm{B}$ 存在但物种 $\mathrm{A}$ 不存在的样方数, $c$ 表示物种 $\mathrm{A}$ 存 在但物种 $\mathrm{B}$ 不存在的样方数, $d$ 表示两个物种都不存 在的样方数, $N$ 为样方总数。通常当 $\chi^{2}>6.635$, 即 $p<$ 0.01 时, 认为种间联结极显著; 当 $3.841<\chi^{2}<6.635$,
即 $0.01<p<0.05$ 时, 认为种间联结显著; 当 $\chi^{2}<$ 3.841 时, 即 $p>0.05$ 时, 认为种间联结不显著。当 $a d<b c$ 时为负联结, $a d>b c$ 时为正联结。

\subsubsection{Ochiai指数测定种间关联程度}

Ochiai指数 $(O I)$ 表示种对的联结性程度和相伴 随机出现的几率, 是一个在 0 与 1 之间变化的无中心 指数, 指数越大, 种对同时出现在样方中的几率越 大。

$$
O I=\frac{a}{(\sqrt{a+b} \times \sqrt{a+c})}
$$

\subsection{5 生态位宽度}

生态位宽度采用Levins生态位宽度计算, 其公 式为:

$$
B_{i}=-\sum_{i=1}^{s} P_{i j} \log P_{i j}
$$

公式(4)中, $B_{i}$ 为物种 $i$ 的生态位宽度; $P_{i j}$ 代表种 $i$ 在第 $j$ 个样方中对资源的利用占全部资源利用的比 例, $P_{i j}=n_{i j} / N_{i j}$, 而 $N_{i j}=\sum n_{i j}$, $n_{i j}$ 为物种 $i$ 在样方 $j$ 中的 重要值, $s$ 为样方数。

\section{2 结果}

\section{1 围栏内外样地主要物种频度、重要值和生态位} 宽度

在围封6年后, 围栏内(表1)的优势种是猪毛蒿、 冷藁、羊草、䊁隐子草、克氏针茅, 与围栏外(表2) 的一致。围栏内外的生态位宽度值排序基本一致, 优势种的生态位排在前面, 说明这几种植物在群落 中分布广泛，对资源的利用能力较强。围栏内的大 籽蒿(Artemisia sieversiana)、冰草(Agropyron cristatum)、瓣莈唐松草(Thalictrum petaloideum)在样方 中的生态位较低, 说明这几种植物对资源的利用处 于劣势地位、资源利用谱低, 对环境的适应能力较 差。

\section{2 群落整体关联性}

公式(1)计算结果表明, 围栏内群落总体关联性 的方差比率值 $V R>1$, 且检验统计量 $\omega$ 没有落入 $\chi^{2}$ 分布给出的界限范围内, 说明 $V R$ 显著偏离 1 。围栏内 群落整体表现出显著的正关联性(表3), 各物种可以 通过对环境因子的优化选择来求得多物种间的稳定 共存。围栏外的比率值 $V R<1$, 检验统计量 $\omega$ 在 $\chi^{2}$ 分 布给出的界限范围内, $V R$ 偏离1不显著。说明围栏外 群落整体不稳定, 存在一定的波动。 
表1 围栏内样地主要物种频度、重要值和生态位宽度

Table 1 The frequency, importance value and niche breadth of species inside the exclosure

\begin{tabular}{|c|c|c|c|c|}
\hline $\begin{array}{l}\text { 编号 } \\
\text { Numbers }\end{array}$ & $\begin{array}{l}\text { 物种 } \\
\text { Species }\end{array}$ & $\begin{array}{c}\text { 频度 } \\
\text { Frequency }\end{array}$ & $\begin{array}{c}\text { 重要值 } \\
\text { Importance value }\end{array}$ & $\begin{array}{c}\text { 生态位宽度 } \\
\text { Niche breadth }\end{array}$ \\
\hline 1 & 猪毛蒿 Artemisia scoparia & 98 & 9.814 & 1.95 \\
\hline 2 & 冷蒿 Artemisia frigida & 84 & 8.608 & 1.88 \\
\hline 3 & 羊草 Leymus chinensis & 91 & 7.747 & 1.93 \\
\hline 4 & 䊅隐子草 Cleistogenes squarrosa & 81 & 6.601 & 1.96 \\
\hline 5 & 克氏针茅 Stipa krylovii & 70 & 5.689 & 1.82 \\
\hline 6 & 花苗宿 Medicago ruthenica & 53 & 5.101 & 1.72 \\
\hline 7 & 碱非 Allium polyrhizum & 67 & 4.243 & 1.81 \\
\hline 8 & 二裂委陵菜 Potentilla bifurca & 45 & 3.965 & 1.47 \\
\hline 9 & 龙蒿 Artemisia dracunculus & 23 & 3.506 & 1.24 \\
\hline 10 & 大籽蒿 Artemisia sieversiana & 8 & 2.581 & 0.86 \\
\hline 11 & 寸草 Carex duriuscula & 15 & 2.553 & 1.14 \\
\hline 12 & 冰草 Agropyron cristatum & 7 & 2.529 & 0.84 \\
\hline 13 & 阿尔泰狗娃花 Aster altaicus & 17 & 2.385 & 1.22 \\
\hline 14 & 瓣芯唐松草 Thalictrum petaloideum & 6 & 2.003 & 0.75 \\
\hline
\end{tabular}

表2 围栏外样地主要物种频度、重要值和生态位宽度

Table 2 The frequency, importance value and niche breadth of species outside the exclosure

\begin{tabular}{|c|c|c|c|c|}
\hline $\begin{array}{l}\text { 编号 } \\
\text { Numbers }\end{array}$ & $\begin{array}{l}\text { 物种 } \\
\text { Species }\end{array}$ & $\begin{array}{c}\text { 频度 } \\
\text { Frequency }\end{array}$ & $\begin{array}{c}\text { 重要值 } \\
\text { Importance value }\end{array}$ & $\begin{array}{c}\text { 生态位宽度 } \\
\text { Niche breadth }\end{array}$ \\
\hline 1 & 鋉隐子草 Cleistogenes squarrosa & 97 & 10.180 & 1.86 \\
\hline 2 & 冷蒿 Artemisia frigida & 79 & 9.674 & 1.84 \\
\hline 3 & 猪毛蒿 Artemisia scoparia & 92 & 8.676 & 1.93 \\
\hline 4 & 羊草 Leymus chinensis & 73 & 6.447 & 1.81 \\
\hline 5 & 克氏针茅 Stipa krylovii & 55 & 6.284 & 1.71 \\
\hline 6 & 碱韭 Allium polyrhizum & 82 & 5.518 & 1.89 \\
\hline 7 & 栉叶蒿 Neopallasia pectinata & 20 & 4.491 & 1.25 \\
\hline 8 & 二裂委陵菜 Potentilla bifurca & 33 & 3.874 & 1.61 \\
\hline 9 & 大籽蒿 Artemisia sieversiana & 20 & 3.657 & 1.29 \\
\hline 10 & 冰草 Agropyron cristatum & 26 & 3.397 & 1.41 \\
\hline 11 & 寸草 Carex duriuscula & 28 & 3.165 & 1.42 \\
\hline 12 & 花苜宿 Medicago ruthenica & 37 & 2.864 & 1.55 \\
\hline 13 & 天名精 Carpesium abrotanoides & 22 & 2.613 & 1.32 \\
\hline 14 & 龙蒿 Artemisia dracunculus & 18 & 2.585 & 1.34 \\
\hline
\end{tabular}

表3 围栏内外群落整体关联性变化

Table 3 Dynamics of overall associations inside and outside the exclosure

\begin{tabular}{lcccl}
\hline & $\begin{array}{c}\text { 方差比率 } \\
\text { Variance ratio }\end{array}$ & $\begin{array}{c}\text { 检验统计量 } \\
\text { Statistic } \omega\end{array}$ & $\begin{array}{c}\chi^{2} \text { 临界值 } \\
\chi^{2} \text { threshold value }\end{array}$ & $\begin{array}{l}\text { 结果 } \\
\text { Results }\end{array}$ \\
\hline 围栏内 Inside the exclosure & 1.320 & 132.42 & {$[77.93,124.34]$} & 显著正联结 Significantly positive association \\
围栏外 Outside the exclosure & 0.931 & 93.11 & {$[77.93,124.34]$} & 不显著负联结 Insignificantly negative association \\
\hline
\end{tabular}

\section{3 围栏内外种间联结性检验}

围栏内外的 $\chi^{2}$ 检验结果(图1，图2)显示，14个物 种共组成 91 个种对, 其中围栏内有 44 个正联结种对 (占总对数的 $48.4 \%$ ) 和 47 个负联结种对 (占总对数的 $51.6 \%)$ 。从显著性结果来看, 正联结显著率(9.9\%)
大于负联结显著率 $(4.4 \%)$ 。围栏外有 47 个正联结种 对(占总对数的 $51.6 \%$ ) 和 44 个负联结种对(占总对数 的 48.4\%)。显著性结果正联结显著率(19.9\%)大于负 联结显著率( $8.8 \%)$ 。

整体比较围栏内外结果发现, 围栏内(图1)优势 


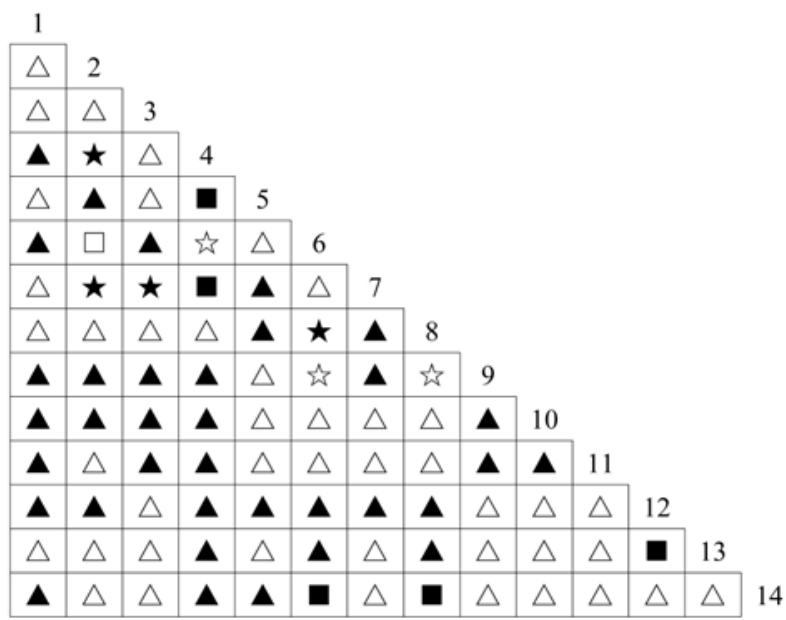

太极显著正联结 Positive correlation at $p=0.01\left(\chi^{2}>6.635\right)$

- 显著正联结 Positive correlation at $p=0.05\left(3.841<\chi^{2}<6.635\right)$

\正联结 Insignificantly positive correlation $\left(\chi^{2}<3.841\right)$

论极显著负联结 Negative correlation at $p=0.01\left(\chi^{2}>6.635\right)$

口显著负联结 Negative correlation at $p=0.05\left(3.841<\chi^{2}<6.635\right)$

$\triangle$ 负联结 Insignificantly negative correlation $\left(\chi^{2}<3.841\right)$

图1 围栏内 $\chi^{2}$ 检验半矩阵图。物种代号: 1 , 猪毛蒿; 2 , 冷蒿; 3, 羊草; 4, 糙隐子草; 5, 克氏针茅; 6, 花苜宿; 7, 碱非; 8, 二裂委陵菜; 9, 龙蒿; 10 , 大籽蒿; 11 , 寸草; 12 , 冰草; 13 , 阿尔泰狗娃花; 14 , 瓣荵唐松草。

Fig. 1 The semi-matrix of the $\chi^{2}$ test inside the exclosure. Species codes: 1, Artemisia scoparia; 2, Artemisia frigid; 3, Leymus chinensis; 4, Cleistogenes squarrosa; 5, Stipa krylovii; 6, Medicago ruthenica; 7, Allium polyrhizum; 8, Potentilla bifurca; 9, Artemisia dracunculus; 10, Artemisia sieversiana; 11, Carex duriuscula; 12, Agropyron cristatum; 13, Aster altaicus; 14, Thalictrum petaloideum.

种和伴生种物种间联结性显著的种对数很少, 大多 数物种间的联结性较弱, 物种的分布和存在受其他 物种的影响很小, 群落发展比较稳定。围栏外(图2), 优势种冷蒿与伴生种栉叶蒿(Neopallasia pectinata)、 二裂委陵菜、大籽蒿、寸草、天名精(Carpesium abrotanoides)之间极显著负相关, 说明冷蒿与这几 个物种生活型差异较大, 种对间的生态位重叠较小, 对环境资源的需求差异也大。而伴生种栉叶蒿、二 裂委陵菜、大籽蒿、冰草 4 个物种间呈极显著正相关, 说明它们对环境具有相似的生态适应性。伴生种与 其他物种之间的联结显著也说明这几个物种的依赖 性强, 在群落中的分布容易受到其他物种的影响且 很不稳定, 在受到外界干扰时容易发生波动。

\section{4 围栏内外群落种间关联程度测定}

从整体上看, $O I$ 大部分小于 0.6 (围栏内占 $78 \%$, 围栏外占 $82 \%$, 表明围栏内外 14 个物种两两间的关

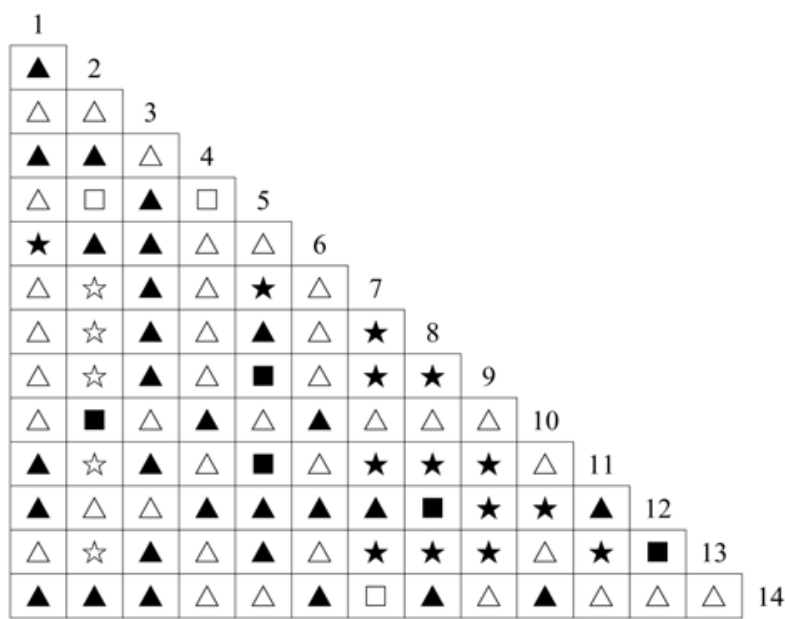

太极显著正联结 Positive correlation at $p=0.01\left(\chi^{2}>6.635\right)$

显著正联结 Positive correlation at $p=0.05\left(3.841<\chi^{2}<6.635\right)$

$\boldsymbol{\Delta}$ 正联结 Insignificantly positive correlation $\left(\chi^{2}<3.841\right)$

极极显著负联结 Negative correlation at $p=0.01\left(\chi^{2}>6.635\right)$

口显著负联结 Negative correlation at $p=0.05\left(3.841<\chi^{2}<6.635\right)$

$\triangle$ 负联结 Insignificantly negative correlation $\left(\chi^{2}<3.841\right)$

图2 围栏外 $\chi^{2}$ 检验半矩阵图。物种代号: 1, 猪毛蒿; 2 , 冷蒿; 3 , 羊草; 4, 粘隐子草; 5, 克氏针茅; 6 , 花苜宿; 7, 碱非; 8, 二裂委陵菜; 9 , 龙蒿; 10 , 大䊏蒿; 11 , 寸草; 12 , 冰草; 13 , 阿尔泰狗娃花; 14 , 瓣莣唐松草。

Fig. 2 The semi-matrix of the $\chi^{2}$ test outside the exclosure. Species codes: 1, Artemisia scoparia; 2, Artemisia frigid; 3 , Leymus chinensis; 4, Cleistogenes squarrosa; 5, Stipa krylovii; 6, Medicago ruthenica; 7, Allium polyrhizum; 8, Potentilla bifurca; 9, Artemisia dracunculus; 10, Artemisia sieversiana; 11, Carex duriuscula; 12, Agropyron cristatum; 13, Aster altaicus; 14, Thalictrum petaloideum.

联程度相对偏弱。围栏内外都表现为优势种猪毛蒿 (Artemisia scoparia)、冷蒿、羊草、粘隐子草、克氏 针茅种间的OI较大, 这就表明这几个物种较强的关 联性; 而伴生种花苜宿、碱非、二裂委陵菜、龙蒿 (Artemisia dracunculus)、冰草等种对间的OI较小, 说明这些物种的关联性较弱, 在群落中同时出现的 几率小, 彼此间相互排斥(表4)。

围栏内的 $\chi^{2}$ 检验中, 呈极显著正关联的种对 $O I$ (图3)都较大, 如种对2-4 (冷蒿-粘隐子草) $O I$ 为 0.93 , 种对 2-7 (冷蒿-碱非) OI为 0.84 , 种对 3-7 (羊草-多根 葱) $O I$ 为 0.85 , 种对 $6-8$ (扁宿豆-二裂委陵菜) $O I$ 为 0.7 ; 而显著负关联的种对 $O I$ 相对较低, 如 4-6 (糙隐 子草-花苜宿) $O I$ 为 $0.55,6-9$ (花苜宿-龙蒿) $O I$ 为 $0.09,8-9$ (二裂委陵菜-龙蒿) $O I$ 为 0.12 。围栏外 (图4) 也表现出相同的趋势，如极显著正关联的种对 1-6 (粘隐子草-碱非) $O I$ 为 $0.92,5-7$ (克氏针茅-栉叶蒿) 
表4 围栏内外Ochiai指数 $(O I)$ 统计表

Table 4 Ochiai indices $(O I)$ of inter-specific associations inside and outside the exclosure

\begin{tabular}{|c|c|c|c|c|c|c|c|c|c|c|}
\hline & \multicolumn{2}{|c|}{$0 \leqslant O I<0.2$} & \multicolumn{2}{|c|}{$0.2 \leqslant O I<0.4$} & \multicolumn{2}{|c|}{$0.4 \leqslant O I<0.6$} & \multicolumn{2}{|c|}{$0.6 \leqslant O I<0.8$} & \multicolumn{2}{|c|}{$0.8 \leqslant O I<1$} \\
\hline & $\begin{array}{l}\text { 种对数 } \\
\text { Species } \\
\text { pairs }\end{array}$ & $\begin{array}{c}\text { 百分比 } \\
\text { Percentage } \\
\text { (\%) }\end{array}$ & $\begin{array}{c}\text { 种对数 } \\
\text { Species } \\
\text { pairs }\end{array}$ & $\begin{array}{c}\text { 百分比 } \\
\text { Percentage } \\
\text { (\%) }\end{array}$ & $\begin{array}{c}\text { 种对数 } \\
\text { Species } \\
\text { pairs }\end{array}$ & $\begin{array}{c}\text { 百分比 } \\
\text { Percentage } \\
(\%)\end{array}$ & $\begin{array}{c}\text { 种对数 } \\
\text { Species } \\
\text { pairs }\end{array}$ & $\begin{array}{c}\text { 百分比 } \\
\text { Percentage } \\
\text { (\%) }\end{array}$ & $\begin{array}{l}\text { 种对数 } \\
\text { Species } \\
\text { pairs }\end{array}$ & $\begin{array}{c}\text { 百分比 } \\
\text { Percentage } \\
\text { (\%) }\end{array}$ \\
\hline 围栏内 Inside the exclosure & 20 & 22 & 36 & 39.6 & 15 & 16.5 & 7 & 7.7 & 13 & 14.3 \\
\hline 围栏外 Outside the exclosure & 8 & 8.8 & 21 & 23.1 & 46 & 50.5 & 8 & 8.8 & 9 & 9.9 \\
\hline
\end{tabular}

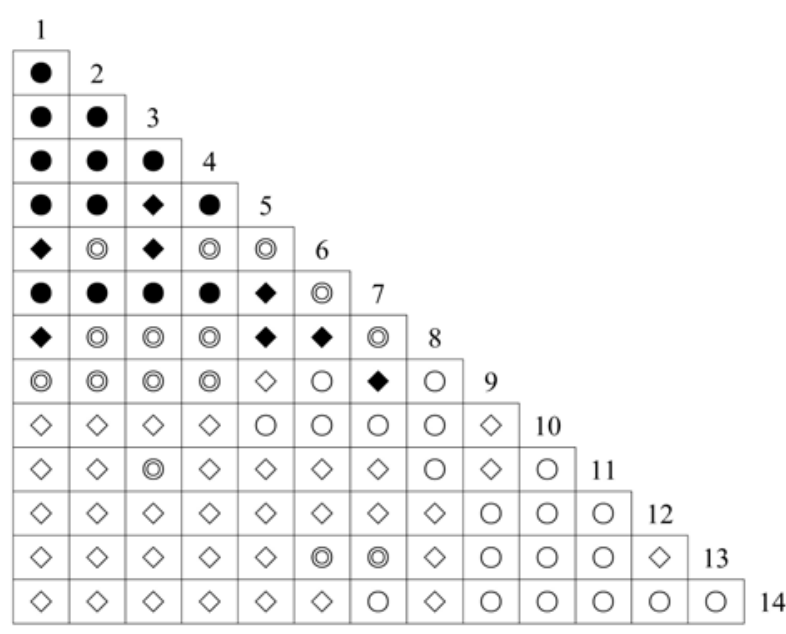

$\circ 0 \leqslant O I<0.2 \diamond 0.2 \leqslant O I<0.4 \quad$ () $0.4 \leqslant O I<0.6$

- $0.6 \leqslant O I<0.8 \bullet 0.8 \leqslant O I<1$

图3 围栏内Ochiai指数 $(O I)$ 半矩阵图。物种代号: 1, 猪毛蒿; 2, 冷蒿; 3, 羊草; 4, 糙隐子草; 5, 克氏针茅; 6, 花苜宿; 7, 碱非; 8 , 二裂委陵菜; 9 , 龙蒿; 10 , 大籽蒿; 11 , 寸草; 12 , 冰 草; 13, 阿尔泰狗娃花; 14 , 瓣荵唐松草。

Fig. 3 The semi-matrix of Ochiai indices $(O I)$ for species in plots inside the exclosure. Species codes: 1, Artemisia scoparia; 2, Artemisia frigid; 3, Leymus chinensis; 4, Cleistogenes squarrosa; 5, Stipa krylovii; 6, Medicago ruthenica; 7, Allium polyrhizum; 8, Potentilla bifurca; 9, Artemisia dracunculus; 10, Artemisia sieversiana; 11, Carex duriuscula; 12, Agropyron cristatum; 13, Aster altaicus; 14, Thalictrum petaloideum.

$O I$ 为 $0.51,7-11$ (栉叶蒿-寸草) $O I$ 为 $0.64,9-11$ (大籽 蒿-寸草) $O I$ 为 0.6 , 极显著负关联的种对 2-7 (冷蒿栉叶蒿) $O I$ 为 $0.23,2-8$ (冷蒿-二裂委陵菜) $O I$ 为 0.37 , 2-9 (冷蒿-大籽蒿) $O I$ 为 $0.22,2-11$ (冷蒿-寸草) $O I$ 为 0.32 , 2-13 (冷蒿-天名精) $O I$ 为 0.24 。

也有一些种对, 如围栏内(图3)的种对1-2 (猪毛 蒿-冷蒿) $O I$ 为 $0.9,2-4$ (冷蒿-䊁隐子草) $O I$ 为 0.93 , 3-7 (羊草-多根葱) $O I$ 为 0.85 ; 围栏外(图4)的1-3 (粘 隐子草-猪毛蒿) $O I$ 为 $0.94,2-4$ (冷蒿-羊草) $O I$ 为 0.8 , 3-6 (猪毛蒿-多根苟) $O I$ 为 0.9 , 这些种对 $\chi^{2}$ 检验中关 联性不显著但 $O I$ 关联指数却相对较高。

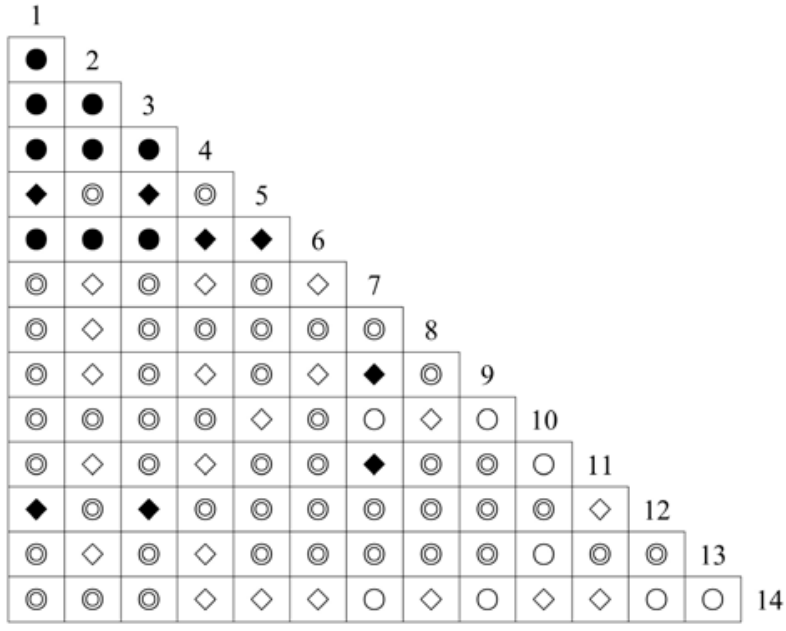

$\circ 0 \leqslant O I<0.2 \diamond 0.2 \leqslant O I<0.4 \quad 0.4 \leqslant O I<0.6$

$\bullet 0.6 \leqslant O I<0.8 \bullet 0.8 \leqslant O I<1$

图4 围栏外Ochiai指数 (OI) 半矩阵图。物种代号: 1, 猪毛蒿; 2, 冷蒿; 3, 羊草; 4, 粈隐子草; 5, 克氏针茅; 6 , 花苜宿; 7 , 碱非; 8 , 二裂委陵菜; 9 , 龙蒿; 10 , 大䊀蒿; 11 , 寸草; 12 , 冰 草; 13 , 阿尔泰狗娃花; 14 , 瓣苾唐松草。

Fig. 4 The semi-matrix of Ochiai indices (OI) for species in plots outside the exclosure. Species codes: 1, Artemisia scoparia; 2, Artemisia frigid; 3, Leymus chinensis; 4, Cleistogenes squarrosa; 5, Stipa krylovii; 6, Medicago ruthenica; 7, Allium polyrhizum; 8, Potentilla bifurca; 9, Artemisia dracunculus; 10, Artemisia sieversiana; 11, Carex duriuscula; 12, Agropyron cristatum; 13, Aster altaicus; 14, Thalictrum petaloideum.

\section{5 围栏内外物种间Spearman秩相关分析}

图5、图6分别是围栏内、围栏外14个物种种间 Spearman半矩阵图, Spearman秩相关分析中，围栏 内 (图5)极显著和显著正相关的有 10 个种对 (占 $10.99 \%$ )，极显著和显著负相关的有 16 个种对(占 $17.58 \%)$; 优势种猪毛蒿、冷蒿、羊草、䊁隐子草、 克氏针茅间大多表现为显著的负相关，这表明这几 个物种对资源的利用方式有较大的差异。而群落整 体联结性较弱, 说明各物种具有较为独立的分布格 局，彼此间受到的影响比较小，群落处于稳定发展 的状态。围栏外(图6)极显著和显著正相关的有 12 个 


\begin{tabular}{|c|c|c|c|c|c|c|c|c|c|c|c|c|}
\hline$-0.41 * *$ & 2 & & & & & & & & & & & \\
\hline $0.21 *$ & $-0.30 * *$ & 3 & & & & & & & & & & \\
\hline-0.83 & $0.22 *$ & $-0.28 *$ & 4 & & & & & & & & & \\
\hline 0.16 & -0.05 & $-0.21 *$ & $0.22 * *$ & 5 & & & & & & & & \\
\hline-0.15 & $-0.21 *$ & 0.17 & $-0.66^{* *}$ & $-0.34 * *$ & 6 & & & & & & & \\
\hline-0.07 & $0.26 * *$ & $-0.22 *$ & $0.29 * *$ & -0.10 & $-0.26 *$ & 7 & & & & & & \\
\hline-0.08 & $-0.24 * *$ & -0.17 & $-0.35^{* *}$ & 0.03 & $0.37 * *$ & -0.05 & 8 & & & & & \\
\hline-0.01 & -0.04 & -0.04 & $0.37 * *$ & 0.10 & -0.41 ** & 0.14 & $-0.27^{*}$ & 9 & & & & \\
\hline-0.12 & -0.05 & -0.05 & -0.05 & -0.15 & -0.02 & $-0.21^{*}$ & -0.05 & 0.08 & 10 & & & \\
\hline-0.09 & -0.07 & 0.04 & 0.01 & 0.03 & -0.11 & -0.07 & 0.02 & 0.01 & 0.07 & 11 & & \\
\hline-0.15 & -0.04 & -0.09 & -0.02 & $0.23 *$ & 0.01 & -0.14 & 0.05 & -0.07 & 0.05 & -0.12 & 12 & \\
\hline$-0.30 * *$ & -0.02 & -0.02 & -0.14 & $-0.25 * *$ & $0.27 * *$ & -0.05 & 0.09 & -0.14 & -0.04 & -0.12 & $0.26 * *$ & 13 \\
\hline-0.19 & -0.02 & -0.12 & -0.03 & -0.11 & 0.12 & -0.19 & 0.17 & -0.14 & -0.07 & -0.11 & 0.09 & -0.02 \\
\hline
\end{tabular}

图5 围栏内14个物种间Spearman相关分析半矩阵图。物种代号: 1, 猪毛蒿; 2, 冷蒿; 3, 羊草; 4, 䊁隐子草; 5, 克氏针茅; 6, 花 $\mathrm{f}$ 苜宿; 7, 碱非; 8 , 二裂委陵菜; 9 , 龙蒿; 10 , 大籽蒿; 11 , 寸草; 12 , 冰草; 13 , 阿尔泰狗娃花; 14 , 瓣芯唐松草。

Fig. 5 The semi-matrix of the Spearman correlation analysis for species in plots inside the exclosure. Species codes: 1, Artemisia scoparia; 2, Artemisia frigid; 3, Leymus chinensis; 4, Cleistogenes squarrosa; 5, Stipa krylovii; 6, Medicago ruthenica; 7, Allium polyrhizum; 8, Potentilla bifurca; 9, Artemisia dracunculus; 10, Artemisia sieversiana; 11, Carex duriuscula; 12, Agropyron cristatum; 13, Aster altaicus; 14, Thalictrum petaloideum.

\begin{tabular}{|c|c|c|c|c|c|c|c|c|c|c|c|c|}
\hline 0.04 & 2 & & & & & & & & & & & \\
\hline 0.05 & $-0.51 * *$ & 3 & & & & & & & & & & \\
\hline 0.05 & $0.20^{*}$ & -0.06 & 4 & & & & & & & & & \\
\hline 0.04 & $-0.32 * *$ & -0.03 & $-0.33 * *$ & 5 & & & & & & & & \\
\hline $0.41 * *$ & $0.20^{*}$ & 0.03 & -0.10 & $-0.39 * *$ & 6 & & & & & & & \\
\hline$-0.45^{* *}$ & $-0.46 * *$ & 0.07 & -0.13 & 0.19 & $-0.40 * *$ & 7 & & & & & & \\
\hline$-0.65^{* *}$ & $-0.57 * *$ & $-0.51^{* *}$ & $-0.27 * *$ & 0.03 & $-0.46^{* *}$ & 0.18 & 8 & & & & & \\
\hline$-0.30^{\text {*** }}$ & $-0.50 * *$ & 0.05 & -0.16 & 0.15 & $-0.38^{* *}$ & $0.43^{* * *}$ & $0.28^{* * *}$ & 9 & & & & \\
\hline-0.07 & $0.30 * *$ & $-0.40^{* *}$ & -0.001 & -0.152 & -0.08 & $-0.21 *$ & -0.11 & -0.07 & 10 & & & \\
\hline$-0.27 * *$ & $-0.47 * *$ & 0.09 & $-0.25 * *$ & 0.16 & $-0.37 * *$ & $0.44 * *$ & $0.32 * *$ & $0.45^{* *}$ & -0.19 & 11 & & \\
\hline-0.19 & 0.02 & $-0.39 * *$ & -0.01 & -0.04 & $-0.22 *$ & 0.13 & 0.18 & 0.18 & $0.33 * *$ & 0.001 & 12 & \\
\hline$-0.76 * *$ & $-0.47 * *$ & $-0.57 * *$ & $-0.33 * *$ & -0.17 & $-0.46^{* *}$ & $0.22^{*}$ & 0.03 & $0.21 *$ & 0.15 & 0.18 & 0.07 & 13 \\
\hline 0.14 & -0.01 & 0.10 & $-0.24 *$ & -0.18 & 0.08 & $-0.23 *$ & 0.01 & -0.11 & -0.01 & 0.03 & -0.14 & -0.19 \\
\hline
\end{tabular}

图6 围栏外 14 个物种间Spearman相关分析半矩阵图。物种代号: 1, 猪毛蒿; 2, 冷蒿; 3, 羊草; 4, 䊁隐子草; 5, 克氏针茅; 6, 花 萛宿; 7, 碱非; 8, 二裂委陵菜; 9, 龙蒿; 10, 大籽蒿; 11, 寸草; 12, 冰草; 13, 阿尔泰狗娃花; 14, 瓣芯唐松草。

Fig. 6 The semi-matrix of the Spearman correlation analysis for species in plots outside the exclosure. Species codes: 1, Artemisia scoparia; 2, Artemisia frigid; 3, Leymus chinensis; 4, Cleistogenes squarrosa; 5, Stipa krylovii; 6, Medicago ruthenica; 7, Allium polyrhizum; 8, Potentilla bifurca; 9, Artemisia dracunculus; 10, Artemisia sieversiana; 11, Carex duriuscula; 12, Agropyron cristatum; 13, Aster altaicus; 14, Thalictrum petaloideum.

种对(占13.19\%), 极显著和显著负相关的有 30 个种 对(占32.97\%); 优势种与伴生种之间呈较为显著的 负相关关系, 说明各物种由于对生境要求的差异而 出现较大的生态位分离, 受外界干扰容易发生波动, 群落处于退化状态。

\section{3 讨论}

\section{1 物种重要值与生态位}

比较发现，围栏内外 14 个物种的重要值并无显 著差异 $(p>0.05)$, 而围栏外冷蒿、糙隐子草、克氏

www.plant-ecology.com 
针茅(表1、表2)的重要值大于围栏内, 这与草本个体 的生物生态学特性相关。汪诗平和王艳芬(2001)的 研究结果发现, 适牧能够刺激粘隐子草个体的地上 净光合效率, 使其在适牧条件下存在超补偿性生长 现象, 超补偿性生长使得粘隐子草能够适应较贫㾑 的生境条件。克氏针茅在中度干扰下也存在植物的 补偿生长效应(赵登亮, 2010)。王静(2003)研究了冷 蒿对放牧干扰的响应机制, 发现随着放牧强度的增 加, 冷蒿的总生物量增加。在放牧干扰下冷高会降 低自身的能量损失, 增加可利用能量, 提高再生长 能力及种群的适合度。

主要优势种猪毛蒿、羊草、冷蒿、克氏针茅等 的生态位宽度大于围栏外的生态位宽度, 而多数伴 生种多根葱、冰草、寸草、二裂委陵菜等的生态位 宽度小于围栏外, 这说明围栏封育保护了主要优势 种的生长。究其原因可能是: 围栏内优势种如猪毛 蒿、克氏针茅、羊草的株高都显著高于伴生种, 且 优势种在群落中分布较为广泛, 使得群落的密闭度 增加, 群落下层个体较小的物种由于对光照等资源 竞争力弱而在群落中减少。如冰草、瓣芯唐松草其 生态位宽度都小于 1 。另外, 自围栏封育后, 围栏内 开始出现零星的小叶锦鸡儿斑块, 轻度放牧和打草 活动会抑制灌木的增加。围栏内由于没有干扰行为, 灌木的盖度和生物量都要大于围栏外。因此围栏内 灌木草本对自然资源、生存空间的竞争比围栏外的 竞争强烈, 这就更加抑制了伴生种的生长。井光花 等(2015)的研究结果表明放牧增加了耐牧物种的生 态位宽度, 因此围栏外的耐牧物种冰草、二裂委陵 菜的生态位宽度要大于围栏内。

\section{2 种间关系分析}

在群落演替发育过程中, 群落中物种间的促进 和干扰作用是同时存在的, 物种之间的净相互作用 是正负相关作用的结果, 但是正负相关作用之间的 平衡点很难掌握, 这与物种生物学特性、环境条件 以及物种的时空分布有关(Aguiar \& Sala, 1994, Callaway et al., 2002)。群落中主要物种间的正负相 关性随着群落发育阶段不断变化。娄彦景和赵鬼义 (2008)认为, 随着群落的演替, 植物各种群之间、种 群与环境之间不断协调, 种内种间的竞争逐渐减小, 个体数量维持稳定, 种对间的联结较为松散, 因此 演替成成熟阶段的植物群落特征表现为优势种之间 没有正联结, 少数发生负联结, 多数为单独分布。太
仆寺旗围封样地内主要物种具有显著相关性的种对 数(26对)占总种对数(91对)的比例偏低, 为 $28.57 \%$, 说明围栏内植物的种间关系比较松散, 种对间的独 立性较强。围栏内优势种猪毛蒿-羊草、猪毛蒿-糙 隐子草之间呈显著正相关关系, 说明猪毛蒿和羊草 与楉隐子草的生态学特性具有互补性, 相伴而生能 促进彼此的生长。而羊草-冷蒿、羊草-䊁隐子草、 羊草-克氏针茅之间呈显著负相关, 说明在围封禁 牧后原有优势种(羊草、冷蒿、粘隐子草、克氏针茅) 出现补偿性生长, 种间对资源和生长空间的竞争加 剧, 彼此相互排斥。另外, 羊草具有很强的营养繁殖 能力, 能够抑制其他草本的生长。

围栏外主要物种间具有显著相关性的种对数 (42对)占总种对数(91对)的比例较高, 为 $46.15 \%$, 说 明围栏外植物的种间关系比较紧密，种对间的依赖 性较强, 一旦受到外界的干扰, 群落容易发生波动。 且负相关的种对较多 (30对，占总对数的 $33.33 \%$ ), 说明围栏外群落中物种之间对资源的竞争较大, 处 于退化阶段。围栏外优势种间呈显著相关性的种对 数不多，优势种间表现出相对松散独立的关系。而 优势种和伴生种之间显著负相关, 说明优势种和伴 生种之间存在较大的种间竞争和干扰。这主要是因 为在家畜的采食和践踏作用下，一方面采食使得适 口性牧草羊草等的生长受到抑制，优势种原本占据 的资源空间得到释放, 使伴生种具有更多的生长空 间。另一方面，践踏使得土壤结构发生变化，使得优 势种和伴生种对土壤资源的竞争加剧。

\section{4 结论}

群落在不同时期出现的动态变化会影响到群落 的功能以及物种间的相互作用。在封育6年后围栏内 群落中优势种之间联结程度高, 同时出现的几率增 大。这一方面可能是由于围封减少了采食以及人为 因素的干扰, 物种生存的外在压力减小。另一方面 是其生态学特性具有互补性, 相伴出现能促进彼此 的生长。而围栏外群落的种间关系较紧密, 彼此间 存在较大的依赖性, 在人为打草和放牧的影响下群 落很容易发生波动, 群落在这些干扰因素的影响下 处于退化状态。围栏封育有利于群落中优势种的 生存。

基金项目 国家自然科学基金(41171445)。 


\section{参考文献}

Aguiar MR, Sala OE (1994). Competition, facilitation, seed distribution and the origin of patches in a Patagonian steppe. Oikos, 70, 26-34.

Callaway RM, Brooker RW, Choler P, Kikvidze Z, Lortie CJ, Michalet R, Paolini L, Pugnaire FI, Newingham B, Aschehoug ET, Armas C, Kikodze D, Cook BJ (2002). Positive interactions among alpine plants increase with stress. Nature, 417, 844-848.

Callaway RM (1996). Competition and facilitation: Contrasting effects of Artemisia tridentata on desert vs montane pines. Ecology, 77, 2130-2141.

Deng ZX, Liu JY, Zhan JY, Zhao T (2004). Dynamic simulation on the spatio-temporal patterns of land use change in Taibus County. Chinese Journal of Plant Ecology, (2), 147-156. (in Chinese with English abstract) [邓祥征, 刘 纪远, 战金艳, 赵涛 (2004). 太仆寺旗土地利用变化时 空格局的动态模拟. 地理研究, (2), 147-156.]

Grechanichenko TE (2001). Changes in the Carabid fauna (Coleoptera, Carabidae) of meadow steppes during the past decades. Russian Journal of Ecology, 32, 117-121.

Henry GHR (1998). Environmental influences on the structure of sedge meadows in the canadian high arctic. Plant Ecology, 134, 119-129.

Jia CXZ, Li SF, Su JR, Su L (2014). Effects of selective logging on inter- and intra-specific interactions of trees in a natural Pinus kesiya forest. Chinese Journal of Plant Ecology, 38, 1296-1306. (in Chinese with English abstract) [贾呈金卓, 李帅锋, 苏建荣, 苏磊 (2014). 择伐 对思茅松天然林乔木种间与种内关系的影响. 植物生 态学报, 38, 1296-1306.]

Jing GH, Cheng JM, Su JS, Wei L, Shi XX, Jin JW (2015). Response of dominant population niche breadths and niche overlaps to various disturbance factors in typical steppe fenced grassland of China's Loess Plateau region. Acta Prataculturae Sinica, 24(9), 43-52. (in Chinese with English abstract) [井光花, 程积民, 苏纪帅, 魏琳, 史晓晓, 金晶炜 (2015). 黄土区长期封育草地优势物种生态位 宽度与生态位重叠对不同干扰的响应特征. 草业学报, 24(9), 43-52.]

Li JB, Ma CP, Lu WH, Zhu JZ, Tursunay, Reyimjan (2009). Effects of enclosing spring-autumn grassland on aboveground biomass at Zhaosu horse ranch. Grassland and Turf, (2), 46-50. (in Chinese with English abstract) [李军 保, 马存平, 鲁为华, 朱进忠, 吐尔逊娜依, 热依木江 (2009). 围栏封育对昭苏马场春秋草地地上植物量的影 响. 草原与草坪, (2), 46-50.]

Li LL, Xu N, Mo XQ, Li HY (2014). Numerical analysis of plants inter-specific relationships in Qilihai wetland. Bulletin of Soil and Water Conservation, 34(4), 70-75. (in Chinese with English abstract) [李兰兰, 许诺, 莫训强,
李洪远 (2014). 七里海湿地植物种间关系的数量分析. 水土保持通报, 34(4), 70-75.]

Li XR (1999). Inter-specific association and correlation of shrub layer in the coniferous-broad leaves mixed geobotanical zone of Russia Plain. Acta Ecologica Sinica, (1), 57-62. (in Chinese with English abstract) [李新荣 (1999). 俄罗斯平原针阔混交林群落的灌木层植物种间相关研 究. 生态学报, (1), 57-62.]

Li ZL, Qin WH, ZHou SB, ZHang JB, Wang Z, Wang YJ (2014). Study on plant niche under fencing measures in the semi-arid grassland of North China. Acta Agrectir Sinica, 22, 1186-1193. (in Chinese with English abstract) [李中 林, 秦卫华, 周守标, 张纪兵, 汪贞, 王运静 (2014). 围 栏封育下华北半干旱草原植物生态位研究. 草地学报, 22, 1186-1193.]

Liu JH, Gao H, Zhang LH, Chen LP, Zhao NX, Gao YB (2010). Comparative analysis of inter-specific association within the Stipa grandis-S. krylovii community in typical steppe of Inner Mongolia, China. Chinese Journal of Plant Ecology, 34, 1016-1024. (in Chinese with English abstract) [刘珏宏, 高慧, 张丽红, 陈丽萍, 赵念席, 高玉 葆 (2010). 内蒙古锡林郭勒草原大针茅-克氏针茅群落 的种间关联特征分析. 植物生态学报, 34, 1016-1024.]

Liu JL (2013). Study on Impact of Mowing and Grazing on Vegetation and Soil of Typical Steppe in Xilingol, Inner Mongolia. PhD dissertation, Chinese Academy of Agricultural Sciences, Beijing. 91. (in Chinese with English abstract) [柳剑丽 (2013). 刈割与放牧对锡林郭勒典型 草原植被和土壤影响的研究. 博士学位论文, 中国农业 科学院, 北京. 91.]

Liu XD, Zhang KB, Wang LL, Yang XH (2015). How enclosure affects community characteristics of the sandy grassland in semi-arid areas of northwestern China. Journal of Beijing Forestry University, 37(2), 48-54. (in Chinese with English abstract) [刘小丹, 张克斌, 王黎黎, 杨晓晖 (2015). 封育对半干旱区沙化草地群落特征的影响. 北 京林业大学学报, 37(2), 48-54.]

Lou YJ, Zhao KY (2008). Analysis inter-specific associations of Carex lasiocarpa community in recent 30-years succession in Sanjiang Plain. Chinese Journal of Ecology, 27, 509-513. (in Chinese with English abstract) [娄彦景, 赵 魁义 (2008). 三江平原毛苔草群落近30年演替过程中 的种间联结性分析. 生态学杂志, 27, 509-513.]

Lü SJ, Liu HM, Wu YL, Wei ZJ, Sun SX, Wang YJ, Lin S (2014). Effect of grazing on niche of constructive and dominant species in the desert steppe. Ecology and Environmental Sciences, 23, 1600-1609. (in Chinese with English abstract) [吕世杰, 刘红梅, 吴艳玲, 卫智军, 孙世贤, 王颖杰, 林森 (2014). 放牧对荒漠草原建群种和优势种 生态位的影响. 生态环境学报, 23, 1600-1609.]

www.plant-ecology.com 
Nathan R (2006). Long-distance dispersal of plants. Science, 313, 786-788.

Nuñez CI, Aizen MA, Ezcurra C (1999). Species associations and nurse plant effects in patches of high-Andean vegetation. Journal of Vegetation Science, 10, 357-364.

Palmer TM, Stanton ML, Young TP (2003). Competition and Coexistence: Exploring mechanisms that restrict and maintain diversity within mutualist guilds. Chicago Journals,162, s63-s79.

Wang J (2003). Studies on Response of Artemisia frigida Population to Grazing Disturbance. PhD dissertation, Mongolia University, Hohhot. 93. (in Chinese with English abstract) [王静 (2003). 冷蒿种群对放牧干扰响应的研究. 博士学位论文, 内蒙古大学, 呼和浩特. 93.]

Wang SP, Wang YF (2001). Study on over-compensation growth of Cleistogenes squarrosa population in Inner Mongolia Steppe. Acta Botanica Sinica, 43, 413-418. (in Chinese with English abstract) [汪诗平, 王艳芬 (2001). 不同放牧率下糙隐子草种群补偿性生长的研究. 植物 学报, 43, 413-418.]

Xu L, Gao Q, Wang YL (2014). Species richness within a six-year slope exclosure in a temperate grassland and its relationship with aboveground biomass. Ecology and Environmental Sciences, 23, 398-405. (in Chinese with English abstract) [徐粒, 高琼, 王亚林 (2014). 围封6年对温 带典型草原坡地物种多样性及其与地上生物量的关系 的影响. 生态环境学报, 23, 398-405.]
Zaal K, Georgi N (1998). Facilitation in subnival vegetation patches. Journal of Vegetation Science, 9, 261-264.

Zhang F, Shangguan TL (2000). Numerical analysis of interspecific relationships in Anelawagnus mollis community in Shanxi. Acta Phytoecologica Sinica, 24, 351-355. (in Chinese with English abstract) [张峰, 上官铁梁 (2000). 山西翅果油树群落种间关系的数量分析. 植物生态学 报, 24, 351-355.]

Zhang JT (2004). Quantitative Ecology. Science Press, Beijing. (in Chinese) [张金屯 (2004). 数量生态学. 科学出版社, 北京.]

Zhang MX, Wang DX, Kang B, Zhang GG, Liu P, Du YL, Yu F (2015). Interspecific associations of dominant plant populations in secondary forest of Pinus armandii in Qinling Mountains. Scientia Silvae Sinicae, 51(1), 12-21. (in Chinese with English abstract) [张明霞, 王得祥, 康冰, 张岗 岗, 刘璞, 杜焰玲, 于飞 (2015). 秦岭华山松天然次生 林优势种群的种间联结性. 林业科学, 51(1), 12-21.]

Zhao DL (2010). Community Ecological Effect of Grazing on Stipa krylovii Steppe. Master degree dissertation, Inner Mongolia University, Hohhot. 54. (in Chinese with English abstract). [赵登亮 (2010). 放牧对克氏针茅草原群 落的生态效应. 硕士学位论文, 内蒙古大学, 呼和浩特. 54.]

责任编委: 李镇清 责任编辑: 王 藏

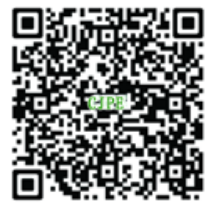

植物生态学报官网

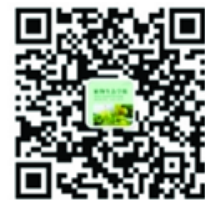

微信订阅号

期刊及学科

相关信息发布

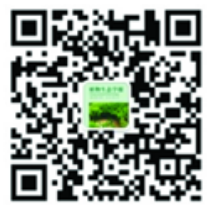

微信服务号

稿件状态查询 全文检索汶览 